\title{
Immunology in the XXI Century - Progress and Achievements
}

\author{
The awarding ceremony for the laureates of the Russian Federation National Award \\ 2011 in the field of science and engineering was held in Moscow this summer. Two \\ interviews with the laureates are published in the popular science section of this \\ issue of Acta Nafurae. Rakhim Musaevich Khaitov, who has been awarded for \\ outstanding achievements in the fundamental and practical development of Russian \\ immunology, talks about the current state of biomedicine.
}

\begin{abstract}
- Rakhim Musaevich, you have been working in immunology for 40 years now. How did it begin?

- It all began in the Samarkand State Medical Institute. Back then, student scientific societies were very popular. I was a member of the radiobiology society.

There was a boom in this field of science at that time; many researchers were interested in the effect radiation has on "living systems." I became interested in the effect of radiation on the immune system. The first books devoted to this subject were just starting to be published. One such book, Immunology of Acute Radiation Injury by R.V. Petrov, left a deep impression on me. When in the radiobiology student society, I started to simulate various immune reactions. I irradiated animals and made attempts at checking whether any antibodies against toxemia products emerged due to irradiation, similar to what occurs in other diseases. I understood back then that there was no way a vaccine against radiation sickness could be designed and that transplantation of bone marrow stem cells was the most adequate approach (the first studies devoted to stem cells were already being carried out). The major challenge was to devise the right method for selecting a donor and a re-
\end{abstract}

cipient based on immune response genes. I became so interested in the subject that I abandoned all other areas of interest and entirely focused on immunology. Unfortunately, there was no immunology in Samarkand in those days. It did not exist as an independent field of science in the USSR. It was a part of virology, microbiology, and infectious disease specialties; just a few hours within various courses and departments were devoted to this subject area. Then I decided to find an institute where I could carry out serious immunological research; so I set off for Moscow, the scientific center of the USSR.

I visited a number of institutes; however, the themes of immunological research were either rather weak or did not sound interesting to me. Finally, I came to the Institute of Biophysics, Ministry of Health of the USSR. They housed the Laboratory of Immunology headed by the already mentioned Professor R.V. Petrov. At the time, he had just brought complex methods of chromosome analysis from England. Those methods were unknown in Russia at the time. The professor suggested that I implement those methods for 3 months. He hinted that if I succeed, I would be hired to work at the laboratory. It took me two weeks to develop them, so I was hired. In just a few months, I had the data published not only in leading journals of the Soviet Union, but in foreign journals, as well.

- You have done great research. What can you say about the condition of the immune system in modern humans and that in humans in the beginning of the $X X$ century? Are there any changes?

- I believe that the immune systems in modern humans and humans who lived in the XIX$\mathrm{XX}$ centuries are fundamentally the same. It is our knowledge and concepts relating to the immune system that have changed. For example, the term "immunity" already existed in the XIX century (the term was proposed by Louis Pasteur and originates from the Latin word "immunitas"); it denoted nonsusceptibility to infectious diseases. Later, in the end of the XIX century, people started to realize that it was not just some kind of abstract nonsusceptibility and that it was associated with particular things (specific proteins and antibodies recognizing bacteria and viruses) and with the cells killing these bacteria and viruses. The immune system remains the same; it is its response to the environment that changes. The system responds to new infections and new 


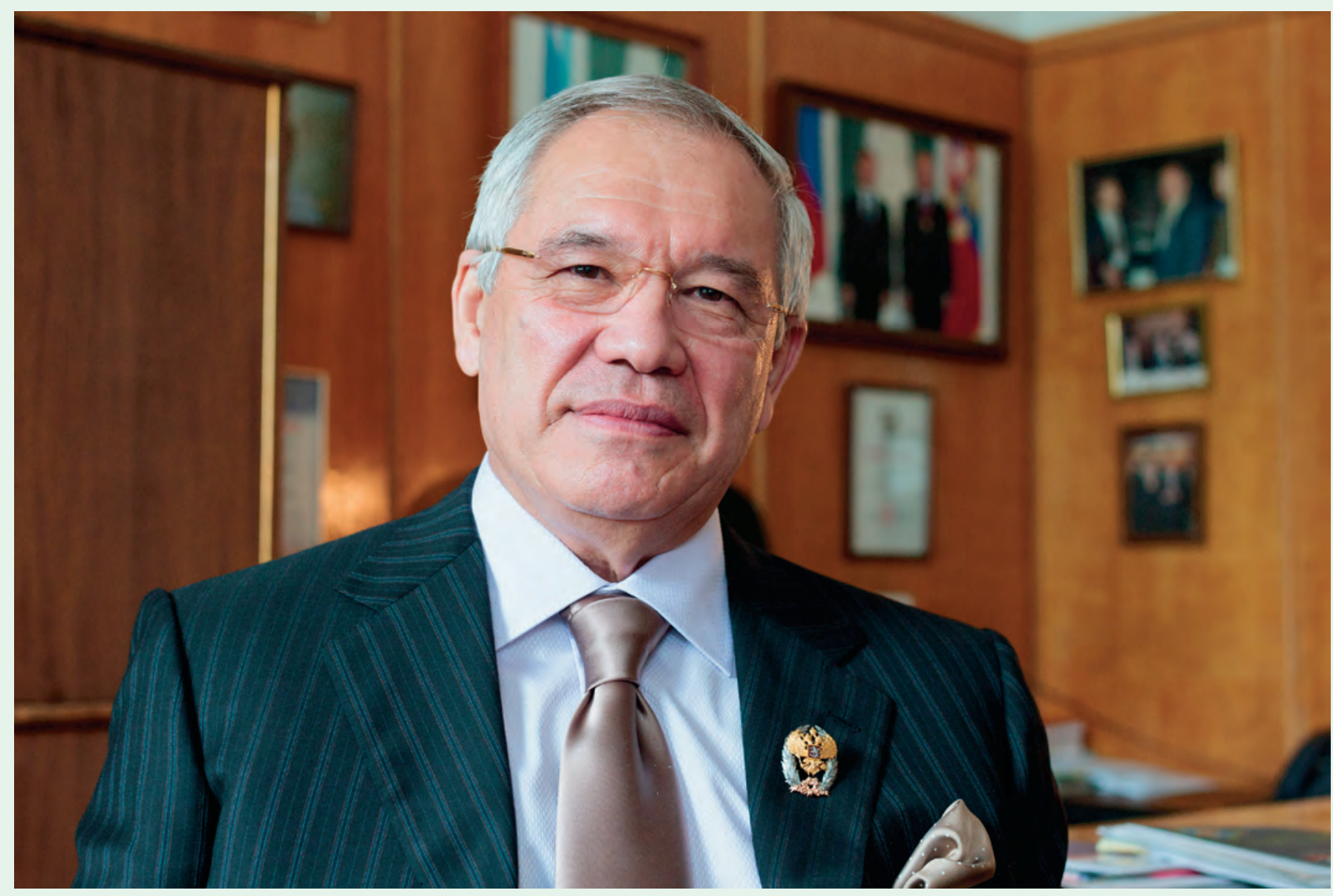

antigens. There are only two systems in the human organism which possess memory and are capable of learning: the nervous and immune systems. Therefore, if an infection did not exist in the XVIII century - for example, the HIV or T-cell leukemia virus - it is natural that the immune system had no cells / proteins that could recognize such foreign elements. These viruses exist today, so the immune system can recognize them.

- An immunological map of Russia has been created on your initiative. Where and how is it used?

- The immune status shows the state of the immune system in the complex. The immune system is very sophisticatedly organized; it includes central and peripheral organs and a large number of

Rakhim Musaevich Khaitov, Director of the Institute of Immunology, Federal Medical-Biological Agency of Russia; Chairman of the Russian Association of Allergology and Clinical Immunology; member of the Council of the European Academy of Allergology and Clinical Immunology; leading expert with the Ministry of Health and Social Development of the Russian Federation for allergology and immunology problems, Academician of the RAS and RAMS, Doctor of Medical Sciences, Professor. Twice-Laureate of the National Award of the Russian Federation and the Russian Federation Government Award.

circulating cells, namely, T-lymphocyte, B-lymphocyte, monocyte, and macrophage families. It is very important that all cells of the immune system interact with each other and are regulated by $\mathrm{T}$ cells (I call them "conductors of the immune system"). There are genes that conduct this immune "orchestra," and it is crucial that all the parameters of the immune system are normal in each person. So we raised the question as to whether the immune system parameters of individuals living in various regions of Russia are identical. For example, a person arrives from the polar region or comes down from the mountains. He can come to our institute and say: "I'm sick. Could you please check my immune system?" And what if the immune system parameters for the region he came from and the new location 
are different? In order to assess the immune status, we established about 120 clinical immunology laboratories in different regions of Russia. The parameters turned out to differ; but this is normal rather than a pathology. In some regions, the amount of T-lymphocytes is higher; in other regions, it is lower. Still other regions are characterized by the highest amount of Blymphocytes. Of course, the differences are small, but they still exist. Thus, it is important to know the parameters of a specific region so that a person knows his/her normal immune status.

- It is a known fact that it is easier to prevent a disease than to cure it. What measures are being taken to prevent immune system disorders?

- There is a national immunization schedule, which includes $N$ vaccines. Immunized children develop nonsusceptibility to these diseases (since the immune system possesses memory). This is one of the measures for fighting an infection. However, there can be genetic pathologies in the immune system; i.e., a child born has congenital disorders of the immune system. The immune system can also be suppressed by such factors as radiation, burns, injuries, a high level of stress, starvation, and exposure to harmful environmental factors. A permanently suppressed immune system results in the development of various disorders: autoimmune diseases, cancer, etc. The immune system is connected to all the other systems in the organism; that is why immune system disorders frequently cause pathologies in a number of other systems in the organism. Therefore, the immune status needs to be checked.

- How can we check it?

- Once you feel there is something wrong with your immune system, you need to take certain measures. What are the first signs?
First of all, they include frequent respiratory tract diseases and other symptoms. In this case, you need to consult an immunologist to determine your immune status. If the immune status is suppressed, immunomodulatory agents are prescribed to reactivate it. We were able to pinpoint the regions where large population groups suffer from immunodeficiency. Preventive immunotherapy was carried out in these groups. It turned out that restoration of the immune system parameters abruptly reduces morbidity.

- Rakhim Musaevich, can the use of immunomodulatory agents be harmful to the organism?

- If your immune system status is fine, there is no need for immunomodulatory agents. One of my articles is even called I Have Never Taken Immunomodulators. Why? I check my immune status once a year. If it is fine, why should I use drugs?

- You mean, there is no need to be overcautious?

- There is no need if your immune status is normal, since it is fraught with autoimmune diseases, can cause allergic reactions, etc. Another situation is if a doctor (I mean not a self-taught doctor, but an immunologist) has diagnosed immune insufficiency. In this case, you need to take immunomodulatory drugs.

- As for allergic diseases, why are so many people susceptible to them nowadays? And a lot of people acquire these diseases in their adulthood...

- You are right. The frequency of allergic diseases has significantly increased recently. In the early XX century, only a negligible percentage of the population suffered from allergies, whereas today this figure is $25-30 \%$ in developed countries. There are regions in Russia where $15 \%$ (and even $30 \%$ ) of the population suffer from allergies. These are extremely high numbers. If you remember, there were a large number of allergic diseases this spring. Spring came early and was warm, so the "green clouds" that emerged made people think about an environmental disaster. In fact, it was abundant blossoming of trees - birch, alder, and hazel trees. The pollen of these trees is responsible for the so-called "seasonal allergy." A lot of people with various symptoms of allergic diseases came for consultations to our institute. Some of them had never had any allergies prior to that. It was the "first wave"; the second wave has already started: other plants and grass are blossoming. There will also be a third wave in autumn.

- And the number of people susceptible to allergies is increasing...

- The point is that the surrounding world is changing. The number of infectious diseases used to be larger and the number of allergic ones smaller. The immune system used to be in a permanent fight against infections, but now we protect children against infections from early childhood. The position of the infections became vacant, so the immune system switched to allergens, which in fact are not infections at all. So apparently harmless proteins cause annoying allergic reactions, which sometimes can be very dangerous. In addition, a great number of triggers of allergy reactions have emerged, including pollutants, exhaust gases, and environmental factors. A number of them are not allergens, but they trigger the development of allergies.

New drugs are constantly coming to market. Most of them are also allergens; therefore, drug allergy is one of the main problems nowadays. Food allergies are rather common: people used to eat traditional food, whereas now a lot of the new foodstuff is produced. In fact, there is no substance today 
that could be called completely allergy-free.

- But some people develop an allergic reaction, whereas others do not...

- Nobody knows why this is a selective process. Scientists know how an allergy develops, what causes it, and what the allergens are. They know the mechanisms of allergy development and how it can be blocked; however, it remains unclear why a person develops an allergy to ovalbumin, while another does not. I think that it is the genes that are responsible for this phenomenon. For example, one person carries genes encoding resistance to ovalbumin, whereas another carries a gene making him susceptible to this allergen and facilitating the development of an allergic reaction. It is possible that once these genes are identified, the allergy problem will be solved.

- Your research group has designed antiallergy drugs. To what extent are you satisfied with the results? Do the drugs have any side effects?

- Yes, antiallergy drugs, allergotropins, have been designed in our institute. These drugs exhibit much higher efficacy compared to the conventional allergens used to cure allergies. No specific side effects have been observed during clinical trials. We have forwarded these medications to the production stage and are awaiting registration and production.

- When is that going to happen?

- That depends on the enterprise that is responsible for processing the documents and obtaining the license for production and application.

- What is the mechanism of action of these drugs?

- First of all, our drugs inhibit the formation of "allergy antibodies." Second, they cause the secretion of protective antibodies, which block the allergic reactions. In other words, we used special molecules to affect different points within the mechanism of allergy development to finally design drugs that are practically significant.

- How do they differ from existing antiallergic agents?

- The already existing drugs the first-, second-, and third-generation antihistamine agents provide conditional treatment of allergies. They inhibit the allergic reaction; so it will return if you stop taking them. Allergen-specific immunotherapy is another type of treatment. What does it consist in? The causal allergen (i.e., the allergen causing a disease) is identified in the patient. The patient then receives the purified form of this allergen for a very prolonged period with the dose increasing gradually. The course lasts $2-3$ months and needs to be repeated during several years. Complete recovery is possible in this case; however, this method is very complex, time-consuming, and fraught with complications. The drugs designed reduce therapy duration to two weeks and 15 injections; they also provide a more than twofold decrease in the need for pharmacotherapy.

- To what extent will this therapy be affordable?

- It is not up to us to decide. I do not think these medications will be more expensive than the allergens used for treatment today.

-An anti-AIDS vaccine is being developed in your institute. What stage are the researchers at?

- It actually is a rather complex problem. It is very difficult to design this vaccine, since the virus mutates quickly. So each patient may host a new variant of the virus. Therefore, many researchers believe that mining for a vaccine is a dead-end approach. However, not all scientists support this point of view. The fact is that even the AIDS virus has conserved regions that do not mutate; therefore, cer- tain proteins or protein fragments in these regions remain intact. So there is a chance that these proteins can be used to design the vaccine. Scientists all over the world are working to solve this problem; however, almost no success has been achieved. Only one American-French group has had some results: two of their vaccines have provided a $35 \%$ reduction in morbidity.

As for me, I believe that another approach should be used in designing the vaccine. The conserved antigens should be combined with immunostimulating agents; this will ensure robust immunity. Since a large number of people have already been infected, there should not only be preventive AIDS vaccines, but therapeutic ones, as well. HIV affects the immune system. Vaccines are inefficient in this case. Therefore, we hope that combination with immunomodulatory agents will ensure a therapeutic effect in infected people and probably even in people with AIDS. Our research group has designed a vaccine of this type; it is known as "Vichrepol."

- What does it consist of?

- We used genetic engineering techniques to design a chimeric protein. This protein contains fragments of various proteins found in the virus (both surface and inner ones, which do not undergo mutation). The protein alone causes no immunological reactions; i.e., it cannot be used as a vaccine. Therefore, it was bound to an immunostimulating agent. This form of the protein had very strong immunostimulating properties. A vast range of preclinical studies and phase I trials in volunteers have been carried out. We are now preparing for phase II trials, which will be more extensive.

- A question emerges: are there any difficulties with clinical trials and recruiting volunteers? 
- The phase I trials need to be carried out only in healthy individuals to make sure that the vaccine is safe and indeed causes two forms of immune response: both antibodies and killers that destroy virusinfected cells are formed. Phase II trials will also be carried out in volunteers. Recruiting volunteers is a very difficult task. Healthy people often do not want to be vaccinated; nevertheless, there are people who are enthusiastic about vaccination (in particular, among the cohorts of sexual minorities).

- What phase of clinical trials is carried out in infected individuals?

- If phase II trials are successful, we will be allowed to proceed to the next phase and include infected people in trials.

- If a vaccine is designed, which population groups will be the first to receive it?

- How was smallpox defeated? Each person was vaccinated with an effective vaccine. There is an influenza vaccine; its effectiveness is lower than that of the smallpox vaccine but still rather high (70$80 \%$ ). Influenza epidemics still occur from time to time. Virus mutation is not the only reason. The fact is that only part of the population has been vaccinated; this includes the so-called "risk group," people who have higher chances to be infected: namely, schoolchildren, children attending kindergartens, doctors, teachers, and public transport employees. If every single person received the vaccine, influenza would presumably be defeated as well. Therefore, with allowance for the enormous scale of the epidemic, if an AIDS vaccine is eventually designed, it will certainly be optimal to vaccinate everyone. A lot of venereal diseases (syphilis and gonorrhea) are widespread today. They are all 100\% curable; however, outbreaks of these diseases happen frequently. The reason is that there is no vaccine against these diseases. Only a vaccine is capable of globally defeating the disease. It is a significant fact that the number of human lives saved thanks to vaccination is much larger than the number saved by medicine as a whole. Vaccination has saved hundreds of millions of people.

- Rakhim Musaevich, how would you estimate the time required to design the vaccine?

- It is difficult to give a prognosis, since there will also be phases III and IV. The latent period of the AIDS virus may be several years (from 2 to $10-20$ years). It is a very sophisticated infection, so it is almost impossible to give any prognoses. What we need to do is to keep working.

- Is the research sufficiently supported by the Government?

- The Government of the Russian Federation has adopted a very good resolution pertaining to the struggle against infectious diseases, including AIDS. Our program has received financial support, which allowed us to design the vaccine. Unfortunately, the resolution has already expired.

- Is the level of Russian research in some areas superior to that of foreign research?

- I would say that Russia is behind Western countries - the U.S., Western European countries, etc. - in terms of the general level of biomedicine. There have been some breakthroughs in Russia, as well. For example, we have done a lot in studying the immune status. Foreign researchers have not dealt with it. We have developed a large number of immunomodulatory agents (dozens agents), whereas only several agents have been designed by foreign researchers. A breakthrough has also been achieved in the area of AIDS vac- cines: we were the first to develop vaccines with immunostimulators. There are no analogues of these vaccines in the world. In the international series AIDS Vaccine Blueprint, which annually reports on promising vaccines, our vaccine is mentioned among the top ten.

In general, there has been a lot of interesting research done, not only in immunology, but in other fields of biomedicine, genetics, molecular genetics, and biochemistry, as well.

- Has the level of AIDS morbidity increased over the past decade in Russia and in the world?

- Unfortunately, it is increasing significantly in Russia. In some countries (such as the U.S., Canada, Western European countries, and Uganda), the increase in the rate of AIDS morbidity was stopped thanks to preventive measures.

- As a research supervisor, can you make special mention of some of your students?

- Quite a few scientists have defended their Candidate or Doctoral thesis papers under my guidance. Now many of them are professors; some of them are even members of Academies of Sciences. Many of my students have received Government and national awards. I do not want to mention any particular names. I respect all of them; they are all good people. If I single out a person or two, the others may be jealous. It is impolite.

- What is your view about further developments in immunology?

- We have a lot of ideas at our institute; in particular, ideas in the fields of molecular immunology and nanoimmunology. We have good financial support and excellent equipment. The only thing that is needed is an inflow of young researchers.

Interview by Maria Morozova 